In zwei Malen vereinigten sich die 'Teilnelımer zu größern gemeinsamen Veranstaltungen, Symposien mit Referaten und ausgedehnter Diskussion über ein zentrales Thema. Zunächst nahm das tropische Afrika, dessen Lebens- und Wirtschaftsbedingungen an verschiedenen Beispielen untersucht und erörtert wurden, die Anwesenden mit Beschlag. Einige Tage später war die Welternährungslage Verhandlungsobjekt. Gerade die Geographen, so meinte Prof. Cressey in einem weitausholenden Vortrag, haben allen Anlaß, sich hierzu zu äußern, können sie doch dank dem Überblick, den sie über den Bedarf an Produkten zufolge der konstanten Bevölkerungsvermehrung auf der Erde und andererseits über die Landnutzungsmöglichkeiten in den einzelnen Kontinenten haben, nicht wenig zur Lösung des so komplexen Problems beitragen, wobei ihnen freilich bei der Erforschung der Grundlagen noch ein weites Feld offensteht. Die Protessoren Schultz (U.S. A.), DE Castro (Brasilien), und Kuriyan (Indien) vervollständigten diese Ausführungen, indem sie auf die besondern Verhältnisse und die damit zusammenhängenden Fragen der Produltionssteigerung in den Vereinigten Staaten, im Amazonasgebiet und in Vorderindien hinwiesen. In der Diskussion ergriff u. a. auch Prof. F. T. WAHLEN (Schweiz), der Leiter der Welternährungsorganisation in Rom, das Wort. Er stellte der optimistischen Betrachtungsweise, die in den meisten Voten vorherrschte, die Bitte gegenüber, vorerst etwas Bedacht zu wahren, denn es sei noch sehr viel Forschungsarbeit zu leisten, zumal hinsichtlich der Nutzungsmöglichkeiten der halbariden Gebiete der Tropen sowie der Ausbeutbarkeit der Wasserkräfte, b:s sich die Wege zu einer guten Lösung des schwierigen Problems erblikken und beschreiten ließen. Einen Vortrag von ebenfalls hohem wissenschaftlichem Gehalt, der freilich zu der späten Stunde, zu der er, gewissermaßen als Abschluß eines ganzen Bankettredenstraußes, gehalten wurde, fehl am Platze war und aus diesem Grunde nicht die Aufmerksamkeit fand, die er ohne Zweifel verdient hätte, hielt endlich Dr. H. W. Ahlmann (Schweden). Er verlas die von ihm verfaßte, traditionelle J. BowmaN-Gedenkschrift, in der er sich ausführlich über die Klimaund die damit zusammenhängenden Gletscherschwankungen innerhalb langer Zeitläufe verbreitete.

Um all die Sitzungen rankte sich ein reiches weiteres Programm, dessen Anlässe, vom Empfang durch das Staatsdepartement und vom offiziellen Bankett bis zu Konzerten, Filmvorführungen und sogar sportlichen Anlässen, den Anwesenden Gelegenheit zum Ausspannen und zu gegenseitigem Gedankenaustausch sowie auch zu Besichtigungen in und um Washington gaben. Nicht unerwähnt mag in diesem Zusammenhang die eindrucksvolle Kartenausstellung bleiben, die von den Vertretern der einzelnen Staaten organisiert wurde und an der die Schweiz mit einer übersichtlichen Darstellung der Entwicklung ihres Kartenwesens ausgezeichnet zur Geltung kam. Die Gruppe der 7 Teilnehmer aus unserm Lande wurde übrigens anläßlich eines Empfangs am Sitz des Schweizer Gesandten von Minister BRUGGMANN und Gattin herzlich willkommen geheißen.

Mit zum Teil mehrwöchigen Exkursionen, welche die Industriegebiete, den Südosten und den Westen der U.S. A. zum Ziele hatten, schloß die in allen Teilen wohlgeglückte Veranstaltung.

\title{
DER IV. INTERNATIONALE KONGRESS FÜR ANTHROPOLOGIE UND ETHNOLOGIE IN WIEN 1952
}

\section{Alfred Steinmann}

Im Vergleich zum vorhergehenden, 1948 in Brüssel abgehaltenen und damals von ungefähr 500 Teilnehmern besuchten III. internationalen Kongreß für Anthropologie und Ethnologie erreichte der IV. vom 1. bis 8. September 1952 in Wien 
tagende Kongreß, an dem über 900 Personen aus 51 Staaten teilnahmen, wiederum Charakter und Umfang der vorkriegsmäßigen Veranstaltungen. Diesen Erfolg verdankt die Tagung der sorgfältigen Planung des Organisationskomitees und seines Präsidenten, Prof. Dr. Wilhelm Schmidt, S. V. D., vor allem aber den unablässigen Bemühungen des Vizepräsidenten, Prof. Dr. Robert von Heine-Geldern und seiner Mitarbeiter, auf deren Schultern die Hauptlast für die Organisation und Durchführung des Kongresses ruhte.

Anwesend waren offizielle Abordnungen der Regierungen von 25 Staaten. Die Schweiz hatte eine aus den HH. Professoren A. Steiniann (Zürich), Marc Sauter (Genf) und Hans Georg Band (Bern) bestehende offizielle Delegation entsandt. Außerdem ließen sich Universitäten, wissenschaftliche Institutionen und Gesellschaften aus 44 Ländern vertreten. Mit Österreich als einladender Nation an der Spitze (40) wurden die umfangreichsten wissenschaftlichen Delegationen von Deutschland (28), Frankreich (26), Großbritannien (25), Italien (23) und die USA (20) gestellt, während diejenigen aus Belgien (17), Holland (14), der Schweiz (14) und Schweden (11) sich ungefähr die Waage hielten. Mit 28 angemeldeten Teilnehmern, die sich folgendermaßen auf die einzelnen Kantone verteilen: Basel 4, Bern 2, Fribourg 3, Genf 2, Neuchâtel 1, Luzern 1, Zürich 15, war die Schweiz überraschend gut vertreten. Überdies waren folgende Delegierte wissenschaftlicher Institutionen und Universitäten aus 7 Städten der Schweiz angemeldet: Basel: Museum für Völkerkunde, Dr. R. Bay, Prof. A. Bühler; Bern: Bernisches historisches Museum, Prof. Dr. H. G. Bandi, Universität, Prof. Dr. H. G. Bandi; Fribourg: Anthropos-Institut, Dr. Fritz Bornemann, Institut d'Etudes Missionaires, Prof. Dr. J.P. Michels; Genève : Société suisse de préhistoire, Université, Prof. Dr. Marc Sauter, Société suisse des Amèricanistes, Dr. Arnold Ith ; Neuchâtel: Société neuchâteloise de Géographie, Prof. Dr. Jean Gabus; Thal-wil: Schweiz. Ges. für Theaterkultur, Dr. Oskar Eberle, Schweizerische Theatersammlung, Dr. Oskar Eberle; Zïrich: Geogr. Ethnographische Gesellschaft, Prof. Dr. A. Steinmann, Sammlung für Völkerkunde der Universität, Dr. Elsy Leuzinger, Schweiz. Gesellschaft für Anthropologie und Ethnologie, Prof. Dr. A. Steinmann.

Eröffnungs- und Schlußsitzung des Kongresses fanden im fahnengeschmückten Auditorium maximum der Universität statt. Bei der Eröffnungsfeier, an der u. a. Unterrichtsminister Dr. KolB sowie Mitglieder des diplomatischen Corps anwesend waren und bei welcher der Rector magnificus der Wiener Universität, Prof. Dr. Alfred Verdross-Drossberg und anschließend der österreichische Bundespräsident Dr. Theodor Körner, der Kongreßpräsident Prof. Dr. Wilhelm Schmint und der Vizepräsident Prof. Dr. Robert von Heine-Geldern Begrüßungsansprachen hielten, konnten die offiziellen Delegierten der Regierungen infolge ihrer großen Anzahl nicht mehr, wie dies noch 1948 beim Kongreß in Brüssel der Fall war, einzeln zur Abhaltung ihrer Begrüßungsreden aufgerufen werden; im Namen der anwesenden Vertreter europäischer Nationen deutscher und französischer Zunge, also auch im Namen der Schweiz, entbot der Delegierte Finnlands, Exz. Dr Ragnar NumEI.IN dem Kongreß Gruß und Dank für die Einladung.

Das wissenschaftliche Programm des Kongresses umfaßte neben allgemeinen Sitzungen, die im Auditorium maximum der Universität abgehalten wurden, solche des Conseil permanent und der einzelnen Sektionen, die teils in den Räumen des Auditorium maximum, teils in verschiedenen Hörsälen der Universität und angegliederten Instituten stattfanden. Von den zahlreichen, in den allgemeinen Sitzungen gehaltenen Vorträgen seien hier nur diejenigen des Kongreßpräsidenten Prof. Dr. W. Schmidt über «Zwei aktuelle Wendepunkte für die Ethnologie», von Dr. A. Métraux (Paris) über «UNESCO and anthropology» mit anschließender Diskussion und der «déclaration de l'UNESCO concernant le problème des races» sowie die von Prof. R. von Heine-Geldern unter dem Titel: «An S. O.S. of ethnology» eingeleitete Besprechung dringender Forschungsaufgaben und der Anregung zur Schaffung einer internationalen Dachorganisation zur Koordinierung der Untersuchungen und ihrer Finanzierung erwähnt, die von allgemeinem Interesse und größerer Tragweite waren. In der Eröffnungssitzung wies Prof. Dr. W. SchMidT auf den sich allmählich vollziehenden Übergang der bisherigen Kolonialvölker zu politisch unabhängigen Nationen, welcher weltgeschichtliche Vorgang sich auf die Völkerforschung in dem Sinne auswirkt, daß diese ehemaligen Kolonialvölker das Studium ihrer eigenen alten Kulturen nun selber auf sich nehmen müssen. Von besonderer Wichtigkeit war auch das höchst aktuelle Thema der A uswertung völkerkundlicher Erkenntnisse für Schule und Volksbildung, wobei speziell auf die Notwendigkeit eines diesbezüglichen Ausbaues des Geschichts- und Geographieunterrichtes hingewiesen wurde. 
Die Abhaltung der Sektionssitzungen, die rund 400 Vorträge und Filmvorführungen umfaßten, erfolgte nach folgendem Plan:

A. 1. allg. Anthropologie; 2. spezielle Anthropologie; 3. Paläanthropologie; 4. biologische Anthropologie.

B. 1a. Geschichte und Methode der Völkerkunde, Soziologie; 1b. Religionswissenschaft; 1c. Kunst, Musik, Tanz; 1d. Kulturpflanzen und Haustiere; 1e. Völkerkunde und Schule. - 2a. Europa; 2b. Nordafrika und vorderer Orient; 2c. Afrika; 2d. Indien; 2e. Ost- und Südstaaten; 2f. Australien und Ozeanien; $2 \mathrm{~g}$. Amerika.

C. Vorgeschichtliche Archäologie und Paläethnologie.

D. Demographie. E. Psychologie. F. Sprachıvissenschaft.

Von den Teilnehmern aus der Schweiz wurden folgende Vortrage gehalten:

BAY, Roland (Basel) „Mesolithische Skelettfunde aus dem Gebiet des Oberrheines und der oberen Donau“. DRExel, A. (Egg bei Zürich) „Die Sprachen der Naturvölker in ihrer Bedeutung für das Problem vom Ursprung der Sprache“, „Die Sprachen Afrikas als Hinweis und Zeugnis für die Wanderung der afrikanischen Völker“, sowie „Bilden die Sprachen Afrikas eine Einheit?“. EberLe, OsKar (Thalwil) „Urtheater (Theater und Urkulturvölker)“, sowie „Die akustische Maske“. Oschinsky, L. (Zürich) „The somatology of the Baganda“. SAuTER, Marc-R. (Genf) „Révision des types raciaux de Chamblandes", sowie "Considérations anthropologiques sur les groupes sanguins en Suisse (ABO et Rh)“ und Schultz, A. (Zürich) "Die relative Dicke der langen Knochen und der Wirbel bei Primaten“.

Ergänzt wurden die wissenschaftlichen Abhandlungen durch Besuche, Vorträge und Demonstrationen in den verschiedenen Museen, von denen beiläufig die Museen für Völkerkunde und Volkskunde, das kunsthistorische Museum, die anthropologischen und prähistorischen Sammlungen im Naturhistorischen Museum, das niederösterreichische Landesmuseum und das österreichische Museum für angewandte Kunst erwähnt seien.

\section{NEKROLOGIE - NÉCROLOGIE}

\section{† GOTTFRIED HURTER (1866-1951)}

An die Namen jener Schweizer, die einen Großteil ihres Lebens in Übersee zubrachten und dank ihres vielseitigen Interesses, ihrer Sammeltätigkeit und ihrer Reisen mit ihren Beobachtungen, Aufzeichnungen und Photos der Forschung wichtiges Material geliefert haben, reiht sich derjenige GOTTFRIED HURTERs würdig an. Z wvar ist der von Jugend auf an Naturwissenschaften und speziell an Vulkanologie, Geographie und Völkerkunde interessierte gebürtige Schaffhauser, der nach Abschluß seiner humanistischen Studien in England und Frankreich eine kaufmännische Lehre absolvierte, 1896 nach Guatemala auswanderte und in Quetzaltenango seine Firma gründete, nie in der Öffentlichkeit hervorgetreten, doch hat er durch seine geographischen Aufzeichnungen der Landschaftstypen Guatemala's, seine Beobachtungen des Ausbruchs und der Gestaltveränderungen des Santa-Maria Vulkans, seine volkskundlichen Studien der Quiché-Indianer und durch sein Interesse für die Kultur der Hochlandmaya, mit denen er zunächst rein geschäftlich in Kontakt gekommen war, manches zur Landes- und Volkskunde von Guatemala beigetragen. Auf seinen seit 1925 gemeinsam mit dem Hamburger Amerikanisten und Ethnologen Prof. Dr. Franz Termer ins Gebiet der Mam-Indianer, in die Umgebung des Atitlan-Sees, in die pazifische Küstenniederung, in die Alta Verapaz und den Izabel-See durchgeführten Reisen konnte er reiches Beobachtungsmaterial zusammenbringen, das er selbstlos Fachgelehrten zur Verfügung stellte. Bei seiner Rückkehr in die Schweiz, im Jahre 1928, ließ sich HURTER nach 30 jähriger Abwesenheit in Frauenfeld nieder, wo er, in Muße seine Aufzeichnungen sammelnd und sichtend, am 5. Dezember 1951 verstorben ist. Seine betagte Schwester, Frau L. HAFFNER-HuRTER, überließ in großzügiger Weise die ansehnliche ethnographische und geographische Bibliothek des Verstorbenen der Sammlung für Völkerkunde und dem geographischen Institut der Zürcher Universität, die ihm ein ehrendes Andenken bewahren wird.

Alfred Steinmann

\section{† ALBERT MONARD (1886-1952)}

Le 27 septembre 1952 est décédé à la Chaux-de-Fonds, à l'âge de soixante-six ans, Alber'T Monard, docteur ès sciences, ancien professeur au Gymnase et directeur du Musée d'histoire naturelle de cette ville.

Le Dr Monard a organisé plusieurs expéditions en Afrique pour récolter et connaitre les faunes d'Angola, de Guinée portugaise et du Cameroun. Chaque fois, à son retour, ce sont des pièces rares qui prennent place au Musée et d'importantes publications paraissent dans les revues scientifiques. Citons, entre autres, son a Voyage de la mission scientifique suisse en Angola 1928- 